\title{
First-passage time: a conception leading to superstatistics
}

\author{
V.V.Ryazanov, S.G.Shpyrko \\ Institute for Nuclear Research, \\ Pr. Nauki, 47, 03068, Kiev, Ukraine
}

Received July 14, 2005

\begin{abstract}
To describe the nonequilibrium states of a system we introduce a new thermodynamic parameter - the lifetime (the first passage time) of a system. The statistical distributions that can be obtained out of the mesoscopic description characterizing the behaviour of a system by specifying the stochastic processes are written. Superstatistics, introduced in [Beck C., Cohen E.G.D., Physica A, 2003, 322A, 267] as fluctuating quantities of intensive thermodynamical parameters, are obtained from statistical distribution with lifetime (random time to system degeneracy) as thermodynamical parameter (and also generalization of superstatistics).
\end{abstract}

Key words: stationary states, stochastic processes, noncanonical distributions, slow dynamics and aging

PACS: $05.70 . \mathrm{Ln}, 05.40 .-\mathrm{a}, 05.10 . \mathrm{Gg}, 05.20 . \mathrm{Gg}$

\section{Introduction} form:

In $[1,2]$ the generalization of Boltzmann factor $\exp \left\{-\beta_{0} E\right\}$ was introduced in the following

$$
B(E)=\int_{0}^{\infty} \mathrm{d} \beta^{\prime} f\left(\beta^{\prime}\right) \mathrm{e}^{-\beta^{\prime} E} .
$$

It is supposed there that the intensive parameter (return temperature $\beta$, chemical potential, etc.) fluctuates. These fluctuations evolve on a long time scale. Locally, in some spatial area (cell) where $\beta$ is approximately constant, the system is described by usual Boltzmann-Gibbs statistics with ordinary Boltzmann factor $\exp \{-\beta E\}$ where $E$ is the energy of a microstate associated with each cell. On a long time scale it is necessary to take into account fluctuations of $\beta$. Superposition of two statistics (that of $\beta$ and that of $\exp \{-\beta E\}$ ) which is therefore referred to as "superstatistics" is derived. This formalism is successfully applied to the description of fully developed hydrodynamic turbulence, defect motion in convection states, the statistics of cosmic rays and other metastable and nonequilibrium phenomena. The special case of these superstatistics, at a function $f$, reduces to gamma-distribution, and appears in the nonextensive statistical mechanics [3,4] describing a number of the physical phenomena which are not satisfactorily described by Boltzmann-Gibbs statistics (for example, long-range many-body systems, systems with memory, numerous phenomena in nuclear physics, astrophysics, geophysics, ecology and other complex systems). In the present work the superstatistics (1) (together with its generalization) is obtained starting with nonequilibrium thermodynamics which being a thermodynamic variable contains a first-passage time, lifetime of statistical system [5-8], section 2 .

Stratonovich in [9] used the term "lifetime" as terminus technicus with respect to the number of phenomena considered. We have encountered the following synonymous expressions: the first passage time (for some given level), escape time, busy period (in the queuing theory) etc. The first published paper on the subject is [10] where the Pontryagin equations for the lifetime distribution are obtained. In 1940 the Kramers work [11] appeared which dealt with the escape time from the

*E-mail: vryazan@kinr.kiev.ua 
potential well. These questions are discussed in books by van Kampen [12], Gardiner [13] and other authors. The lifetime plays a part in the theory of phase transitions, chemical reactions, in investigating the dynamics of the complex biomolecules, in calculating the coefficient of the surface diffusion in semiconductors, in nuclei, elementary particles, spin glasses, spectroscopy, trap systems, in the theory of metastable states etc.

The lifetime is the time period till the moment of the first (random) reattachment of a certain level (for example, zero level) by a random process $y(t)$ for the macroscopic parameter (2) (for example, energy or particle number). The lifetime is the slave process in the terminology of the random processes theory; that of $y(t)$ determines the behaviour of lifetime. The lifetime depends on the energy of a system, its size, fluxes of energy. Therefore a system exchanges the energy, the particle number with the thermostat, but not the "lifetime". The lifetime $\Gamma$ is a macroparameter characterizing the system and its interaction with the environment. It is an observable, well-defined and physically well understandable quantity reflecting important system peculiarities.

In [14] it is pointed out that a nonequilibrium distribution is characterized by an additional macroparameter in the description. One can show [15] that in the method of nonequilibrium statistical operator $(N S O)[16,17]$ this parameter is the time $t-t_{0}$, starting from the birth of a system. In other words this is the first-passage time of the zero level if we inverse the time, the age of the system (in Zubarev work [17] the $N S O$ is obtained by averaging over the initial time; in [18] it is noted that the $\varepsilon$ value from $N S O$ in $[16,17]$ is inversely proportional to the lifetime). In works by Kirkwood [19] it was noted that the state of a system at the current time depends on the whole evolution history of the nonequilibrium processes in it, hence, on its age as well. Therefore, we choose the random lifetime as an additional thermodynamical parameter.

It is worth noting that the notion of lifetime has many interpretations, and its physical applications are also various. For example, the relaxation times can be considered to be lifetimes of the excited states. An analogy can be traced between the approach of the present work and the description of nonergodicity in the spin glasses [20] (although the "lifetime" term was not used there), which included the memory effects (the dependence on the previous history of a system), the dependence on the initial time moment, and on the times of transition between different valleys on the energy profile. This problem is related to the response functions in systems with excited degrees of freedom [21]. In our work we presented the lifetime, like it was done in the pioneering work [10], from the outset in a strict mathematical form (2), similarly to the same value introduced in the theory of random processes. One can admit various physical interpretations of the general mathematical description. One of these possibilities applied in this work to the lifetime of the system in the energetic valleys, leads to the obtained class of statistical distributions containing the product of Gibbs-like factor and that of superstatistics.

In the present work we consider the open nonequilibrium systems, stationary nonequilibrium systems, certain point metastable states. Work on spin glasses and other aging systems, where a "waiting time" plays an important role, points in this direction.

\section{System lifetime and lifetime distribution}

The lifetime $\Gamma_{x}$ (or $\Gamma$ ) is thus a random process which is subordinate (in terms of the definitions of the theory of random processes [22]) with respect to the master process $y(t)$,

$$
\Gamma_{x}=\inf \{t: y(t)=0\}, \quad y(0)=x>0 .
$$

This definition of the lifetime is taken from the theory of random processes where it is widely used in the theory of queues, stochastic theory of storage [23]. We use below as $y(t)$ the value of full energy of the system $E$, assuming its nonnegativity though it is possible to consider $y(t)$ to be the number of particles in the system as well.

Gibbs equilibrium distribution for microcanonic probability density in phase space $z$ corresponds to a condition of equiprobability of all possible microstates compatible with the given value of a macrovariable. We shall assume, that transition of the system in a nonequilibrium condition breaks the equality of probabilities, characteristically for an equilibrium case. One introduces 
an additional observable macroparameter, thus extending the phase space (containing additional degenerate, absorbing states). We shall assume the validity of a principle of equal probabilities for the extended phase space divided into cells with constant values $(E, \Gamma)$ (instead of phase cells with constant values $E)$. The standard procedure (e.g. $[24,32])$ allows one to write down the relation between the distribution density $p(E, \Gamma)=p_{E \Gamma}(x, y)$ and microscopic (coarse-grained) density $\rho(z ; E, \Gamma)$

$$
p(E, \Gamma)=\int \delta(E-E(z)) \delta(\Gamma-\Gamma(z)) \rho(z ; E, \Gamma) \mathrm{d} z=\rho(z ; E, \Gamma) \omega(E, \Gamma) .
$$

The structure factor $\omega(E)$ is thus replaced by $\omega(E, \Gamma)$ - the volume of the hyperspace containing given values of $E$ and $\Gamma$. If $\mu(E, \Gamma)$ is the number of states in the phase space which have the values of $E$ and $\Gamma$ less than given numbers, then $\omega(E, \Gamma)=\mathrm{d}^{2} \mu(E, \Gamma) / \mathrm{d} E \mathrm{~d} \Gamma$. It is evident that $\int \omega(E, \Gamma=y) \mathrm{d} y=\omega(E)$. The number of phase points between $E, E+\mathrm{d} E ; \Gamma, \Gamma+\mathrm{d} \Gamma$ equals $\omega(E, \Gamma) \mathrm{d} E \mathrm{~d} \Gamma$. We make use now of the principle of equiprobability applied to the extended cells $(E, \Gamma)$.

Using a maximum-entropy principle [25] (with Shennon's measure), it is possible to write down an expression for microscopic (but coarse-grained) density of probability in the extended phase space

$$
\rho(z ; E, \Gamma)=\mathrm{e}^{-\beta E-\gamma \Gamma} / Z(\beta, \gamma)
$$

where

$$
Z(\beta, \gamma)=\int \mathrm{e}^{-\beta E-\gamma \Gamma} \mathrm{d} z=\iint \mathrm{e}^{-\beta E-\gamma \Gamma} \omega(E, \Gamma) \mathrm{d} E \mathrm{~d} \Gamma
$$

is the partition function, $\beta$ and $\gamma$ are Lagrange multipliers satisfying the equations for the averages

$$
\langle E\rangle=-\partial \ln Z /\left.\partial \beta\right|_{\gamma} ; \quad\langle\Gamma\rangle=-\partial \ln Z /\left.\partial \gamma\right|_{\beta} .
$$

Introducing $\Gamma$ means more information effectively taken into account than merely in linear terms of the canonical distribution $\exp \{-\beta E\} / Z(\beta)$. The value $\gamma$ thermodynamically conjugated to the lifetime is related to the entropy fluxes and entropy production which characterize the peculiarities of the nonequilibrium processes in an open thermodynamic system. If $\gamma=0$ and $\beta=\beta_{0}=\left(k_{\mathrm{B}} T_{\mathrm{eq}}\right)^{-1}$, where $k_{\mathrm{B}}$ is the Boltzmann constant, $T_{\mathrm{eq}}$ is the equilibrium temperature, then the expressions (4), (5) yield the equilibrium Gibbs distribution. One can thus consider (4)(5) as a generalization of the Gibbs statistics to cover the nonequilibrium situation. The physical phenomena such as metastability, phase transitions, stationary nonequilibrium states are known to violate the equiprobability of the phase space points. The value $\gamma$ can be regarded as a measure of deviation from the equiprobability hypothesis. The value of $\gamma=0$ characterizes equilibrium isolated systems, presenting an idealization. If the detailed balance is satisfied, then in equilibrium $\gamma \neq 0$. Mathematically introducing lifetime means acquiring additional information regarding the underlying stochastic process, beyond merely the knowledge of its stationary distribution, exploring the (stationary) properties of its slave process.

Let us underline the principal features of the suggested approach.

1. We introduce a novel variable $\Gamma$ which can be used to derive additional information regarding the system in a stationary nonequilibrium state. We suppose that $\Gamma$ is a measurable quantity at macroscopic the level, thus values like entropy which are related to the order parameter (principal macroscopic variable) can be defined. At the mesoscopic level the variable $\Gamma$ is introduced as a variable with operational characteristics of a random process slave with respect to the process describing the order parameter.

2. We suppose that thermodynamic forces $\gamma$ related to the novel variable can be defined. One can introduce the "equations of state" $\beta(\langle E\rangle,\langle\Gamma\rangle), \gamma(\langle E\rangle,\langle\Gamma\rangle)$. Thus we introduce the mapping (at least approximate) of the external restrictions on the point in the plane $\beta, \gamma$.

3. We suppose that a "refined" structure factor $\omega(E, \Gamma)$ can be introduced which satisfies the condition $\int \omega(E, \Gamma=y) \mathrm{d} y=\omega(E)$ (ordinary structure factor). This function (like $\omega(E)$ ) 
is the internal (inherent) property of a system. At the mesoscopic level we can ascribe to this function some random process inherent to the system (at given restrictions $\left(\beta_{0}, \gamma_{0}\right)$ ). The structure factor has the meaning of the joint probability density for the values $E, \Gamma$ understood as the stationary distribution of this process.

4. It is supposed that at least for certain classes of effects the resulting distribution has the form (4), (5), i.e., the change of the principal random process belongs to some class of invariance leading to this distribution which explains how one can pass from the process in the reper point $\left(\beta_{0}, \gamma_{0}\right)$ (for example, in equilibrium when $\gamma=0$ and $\beta=1 / k_{\mathrm{B}} T$ ) to a system in an arbitrary nonequilibrium stationary state. The thermodynamic forces should be chosen so that the distribution leads to new (measurable) values of $(\langle E\rangle,\langle\Gamma\rangle)$.

\section{Distribution for indisturbed lifetime}

The use of a specific lifetime distribution depends on the physical peculiarities of the system under investigation. Further detailed elaboration will require the concrete definition of the $\Gamma$ distribution and the interpretation of the Lagrange parameters $\beta$ and $\gamma$. The Lagrange parameter $\beta$ is supposed to be (like the equilibrium Gibbs statistics) as follows:

$$
\beta=1 / k_{\mathrm{B}} T
$$

where $T$ is the average (over the body volume) local equilibrium temperature. Since at fluxes $\vec{q} \neq 0$ the temperature is not the same all over the bulk of the body, one can define $T$ in a system with volume $V$ as the volume average, i.e.

$$
T=V^{-1} \int_{V} T(r, t) \mathrm{d} r
$$

(the same definition was used in [24]). Of course, the thermodynamic description itself is supposed to be already coarse-grained. To get the explicit form of the $\Gamma$ distribution we shall use the general results of the mathematical theory of phase coarsening of the complex systems [26], which imply the following density of distribution of the lifetime for coarsened random process (see also [9]):

$$
p(\Gamma<y)=\Gamma_{0}^{-1} \exp \left(-y / \Gamma_{0}\right)
$$

for one class of the stable states and the Erlang density

$$
p(\Gamma<y)=\sum_{k=1}^{n} R_{k} \Gamma_{0 k}^{-1} \exp \left(-y / \Gamma_{0 k}\right) ; \quad \sum_{k=1}^{n} R_{k}=1
$$

in the case of several $(n)$ classes of the ergodic stable states, $R_{k}$ - probability that the system is in the $k$-th class. The values $\Gamma_{0}$ and $\Gamma_{0 i}$ are averaging of the residence times and the degeneracy probabilities over stationary ergodic distributions (in our case - Gibbs distributions). The physical reason for the realization of the distribution in the form (8), (9) is the existence of the weak ergodicity in a system. Mixing the system states at big times will lead to the distributions (8), (9). As we note in Section 2, the structure factor $\omega(E, \Gamma)$ has a meaning of the joint probability density of values $E, \Gamma$. For the distributions $(9),(10)$ the functions $\omega(E, \Gamma)$ from $(3),(5)$ take on the form:

$$
\omega(E, \Gamma)=\omega(E) \Gamma_{0}^{-1} \exp \left(-\Gamma / \Gamma_{0}\right) ; \quad \omega(E, \Gamma)=\omega(E) \sum_{k=1}^{n} R_{k} \Gamma_{0 k}^{-1} \exp \left(-\Gamma / \Gamma_{0 k}\right)
$$

for the relations (9) and (10) respectively. Substituting into the partition function yields

$$
Z(\beta, \gamma)=Z(\beta)\left(1+\gamma \Gamma_{0}\right)^{-1} ; \quad Z(\beta, \gamma)=Z(\beta) \sum_{k=1}^{n} R_{k}\left(1+\gamma \Gamma_{0 k}\right)^{-1}
$$


for (8) and (9), where $\mathrm{Z}(\beta)=\int \omega(E) \exp (-\beta E) \mathrm{d} E$ is the Gibbs partition function.

Let us note that the value similar to $\gamma$ is defined in [27-29] for fractal objects. It is equal to zero for the closed system, and for open system it is equal to $\Sigma \lambda_{i}-\lambda_{\mathrm{KS}}$, where $\lambda_{i}$ are Lyapunov's parameters, and $\lambda_{\mathrm{KS}}$ is Kolmogorov-Sinai entropy.

\section{Superstatistics from distribution of the kind (5)}

In the distribution (4), (5) containing lifetime, as thermodynamic parameter, joint probability for values $E$ and $\Gamma$ is equal to

$$
\begin{aligned}
p(E, \Gamma) & =\mathrm{e}^{-\beta E-\gamma \Gamma} \omega(E, \Gamma) / Z(\beta, \gamma) \\
Z(\beta, \gamma) & =\int \mathrm{e}^{-\beta E-\gamma \Gamma} \mathrm{d} z=\iint \mathrm{e}^{-\beta E-\gamma \Gamma} \omega(E, \Gamma) \mathrm{d} E \mathrm{~d} \Gamma
\end{aligned}
$$

Having integrated (14) on $\Gamma$, we obtain distribution of a kind

$$
p(E)=\int p(E, \Gamma) \mathrm{d} \Gamma=\exp (-\beta E) \int_{0}^{\infty} \exp (-\gamma \Gamma) \omega(E, \Gamma) \mathrm{d} \Gamma / Z(\beta, \gamma) .
$$

We shall write down

$$
\omega(E, \Gamma)=\omega(E) \omega_{1}(E, \Gamma)=\omega(E) \sum_{k=1}^{n} R_{k} f_{k}(\Gamma, E) .
$$

In the last equality (14) it is supposed that there exist $n$ classes of ergodic states in a system; $R_{k}$ is the probability that the system will be in $k$-th class of ergodic states, $f_{k}(\Gamma, E)$ is density of distribution of lifetime $\Gamma$ in this class of ergodic states (generally $f_{k}$ depends on $E$ ). As physical example of such situation (characteristic of metals, glasses) one can mention the potential of many complex systems. Such a situation is considered in [30]. Minima of potential correspond to the metastable phases, disproportionate structures, etc. Expression (14) is applicable to the description of such systems.

We shall assume an obvious kind of distribution $f_{k}$ in (14), having chosen it as gammadistribution

$$
\begin{aligned}
& f_{k}(x)=x^{\alpha_{k}-1} \mathrm{e}^{-x / b_{k}} / \Gamma\left(\alpha_{k}\right) b_{k}^{\alpha_{k}}, \quad x>0, \quad f_{k}(x)=0, \quad x<0 \\
& \int_{0}^{\infty} \mathrm{e}^{-\gamma_{k} x} f_{k}(x) \mathrm{d} x=\left(1+\gamma_{k} b_{k}\right)^{-\alpha_{k}},
\end{aligned}
$$

$\left(\Gamma(\alpha)\right.$ is gamma-function). We assume that in $k$-th metastable area $\gamma=\gamma_{k}$. We shall choose $\alpha_{k}=\gamma_{k} / \lambda_{k} ; b_{k}=\Gamma_{0 k} \lambda_{k} / \gamma_{k}, \lambda_{k}$ is the intensity of energy flow in the system (subsystem), equal in dynamical equilibrium of an output intensity [31]. For realistic metastable systems the detailed balance principle is satisfied and $\lambda \neq 0$. Then $b_{k} \alpha_{k}=\Gamma_{0 k},\left(1+\gamma_{k} b_{k}\right)^{-\alpha_{k}}=\left(1+\lambda_{k} \Gamma_{0 k}\right)^{-\gamma_{k} / \lambda_{k}} ; \Gamma_{0 k}$ is equal to average lifetime in $k$-th class of metastable states without disturbance from (8)-(11). We understand the absence of stationary forces and the determined fluxes as undisturbed lifetime $\Gamma_{0 k}$; at the system (open metastable area) there are only stochastic fluxes. For average lifetime $\Gamma_{0 k}$ of the system in dynamical equilibrium (or in stationary) state, where at the system with grand canonical sum $Q_{k}$ there are only stochastic fluxes, in work [31] by means of stochastic models of storage it is obtained

$$
\Gamma_{0 k}=\left(Q_{k}-1\right) / \lambda_{k} ; \quad 1+\lambda_{k} \Gamma_{0 k}=Q_{k},
$$

where $Q_{k}=\exp \left\{\beta_{k} P_{k} V_{k}\right\}$ is the grand statistical sum of the grand canonical ensemble of the part of system in $k$-th metastable state, in the $k$-th potential well, $\beta_{k}=\left(1 / k_{\mathrm{B}} T\right)_{k}$ is reverse temperature in $k$-th metastable state, $P_{k}, V_{k}, T_{k}$ are pressure, volume, temperature in $k$-th metastable state, $V$ is the full system volume. 
From (15)-(16) it is seen that

$$
\int_{0}^{\infty} \mathrm{e}^{-\gamma x} f_{k}(x) \mathrm{d} x=\left(1+\lambda_{k} \Gamma_{0 k}\right)^{-\alpha_{k}}=Q_{k}^{-\alpha_{k}}=\mathrm{e}^{-\alpha_{k}\left(P / k_{\mathrm{B}} T\right)_{k} V_{k}} ; \quad \alpha_{k}=\gamma_{k} / \lambda_{k} .
$$

For exponential distribution $f_{k}$ in (15) $\alpha_{k}=1$. Substitution (14), (17) in (13) gives

$$
p(E)=\mathrm{e}^{-\beta E} \omega(E) Z^{-1} \sum_{k=1}^{n} R_{k} \mathrm{e}^{-\beta_{k}\left(P_{k} v_{k} / u\right) \alpha_{k} E},
$$

where $u=E / V$ is specific energy, $v_{k}=V_{k} / V$. At such an approach which can be considered as a discretized version of superstatistics $[1,2]$, one accounts not only for the inverse temperature fluctuations (that of $\beta$ ), but also for the fluctuations of values $P, v, \alpha$. Denote in $(18)\left(P_{k} v_{k} \alpha_{k}\right) / u=$ $r_{k}$ and rewrite the exponent in (18) as $y_{k} \beta_{0} r_{0} E$, where $\beta_{0}=\Sigma R_{k} \beta_{k} \approx \Sigma \beta_{k} / n, r_{0}=\Sigma R_{k} r_{k} \approx \Sigma r_{k} / n$ are averages of $\beta_{k}$ and $r_{k} ; y_{k}=\left(\beta_{k} r_{k} / \beta_{0} r_{0}\right)$. It will be shown below that $r_{0}$ is related to the controlling parameter of a problem (for example, with the feedback coefficient in the Van der Pol generator [24], the birth parameter in the Maltus-Ferhuelst process etc). Since $R_{k}$ is the probability that the system will be in $k$-th class of ergodic states, then $R_{k}$ is the probability that the quantity $y=\left(\beta r / \beta_{0} r_{0}\right)$ will take on the value $y_{k}=\left(\beta_{k} r_{k} / \beta_{0} r_{0}\right) ; R_{k}=R\left(y=y_{k}\right)$. Thus the expression under sum in r.h.s. of (19) is the characteristic function $\varphi(t)=\sum_{i=1}^{n} \mathrm{e}^{i t x_{i}} R_{i}$ of the distribution $R_{k}$ with the argument $i t=-\beta_{0} r_{0} E$. If $R_{k}=\delta_{k 0}$, where for a cell with zero index $\beta_{k} r_{k}=\beta_{0} r_{0}$, then (18) acquires the form $p(E) \sim \exp \left(-\beta E-\beta_{0} r_{0} E\right)=\exp \left(-\beta_{0} E\right)$, where the open character of the system (the existence of fluxes) is taken into account, therefore the inverse temperature is set to be not $\beta=1 / k T$, but $\beta_{0}$. Thus the relation between $\beta$ and $\beta_{0}$ is $\beta=\beta_{0}\left(1-r_{0}\right) ; \beta_{0}=\beta /\left(1-r_{0}\right)$.

We shall write down (18) in the form

$$
p(E)=\mathrm{e}^{-\beta E} \omega(E) \varphi(E) / Z_{A} ; \quad \varphi(t)=\sum_{i=1}^{n} \mathrm{e}^{i t x_{i}} R_{i} ; \quad \text { it }=-\beta_{0} r_{0} E
$$

and consider different resources of the task of function $\varphi(E)$ in (19). Let us choose as $R_{k}$ in (19) the probabilities of the negative binominal distribution (a discrete analogue of the continuous gamma distribution) with a characteristic function $\varphi(t)=\sum_{i=1}^{n} \mathrm{e}^{i t x_{i}} R_{i}=[p /(1-(1-p) \exp (i t))]^{r}, r$ and $p$ being parameters. Setting $r=1 /(q-1)$, it $=-\beta_{0} r_{0} E, p=1 /[1+(q-1)]=1 / q$, we conclude that (19) in this case is

$$
p(E) \sim \mathrm{e}^{-\beta E}\left[1+(q-1)\left(1-\mathrm{e}^{-\beta_{0} r_{0} E}\right)\right]^{-1 /(q-1)} .
$$

In this approach the behaviour of $p(E)$ is asymptotically not power-like, as in Tsallis [3,4] distributions, but rather exponential. The argument $\beta_{0} r_{0} E$ in the Tsallis distribution [3,4] is replaced by $\left(1-\mathrm{e}^{-\beta_{0} r_{0} E}\right)$. As $R_{k}$ one can choose various specific forms of distributions.

As example of application of expressions (4)-(6), (12)-(14) can consider a flow of rarefied gas in a tube of radius $R$ and lengths $L, L \gg R$. By use of (8)-(11), (15) and storage model [31] we obtain

$$
p(E)=\mathrm{e}^{-\beta E} \omega(E) / Z\left[1-2 R(1 / 2 m)^{1 / 2}\left(n_{1}-n_{2}\right)\left(k_{\mathrm{B}} E / C_{\mathrm{V}}\right)^{1 / 2} / 3 \pi L a_{f}\right],
$$

where $E$ is microscopic random internal energy, $m$ is weight of atoms, $n_{1}, n_{2}$ are densities of gas on the ends of a tube, $C_{\mathrm{V}}$ is a thermal capacity of system at a constant volume, value $a_{f}=$ const can be defined as the maximal value of a flux, at which there exist stationary states in laminar mode, flux in a tube is $q=2 R\left(m / 2 k_{\mathrm{B}} T\right)^{1 / 2}\left(P_{1}-P_{2}\right) / 3 \pi L=2 R(1 / 2 m)^{1 / 2}\left(n_{1}-n_{2}\right)\left(k_{\mathrm{B}} U / C_{\mathrm{V}}\right)^{1 / 2} / 3 \pi L$ [32]. Value $q=a_{f}$ is a point of nonequilibrium phase transition from a laminar mode to turbulent. As another example we shall consider the system of neutrons in a nuclear reactor and we shall obtain distribution of energy of neutrons in a nuclear reactor in view of finiteness of their lifetime. 


\section{Continuos approximation for the superstatistics factor}

If in expressions (18)-(19) we denoted $r_{1}=P^{\prime} v^{\prime} \alpha^{\prime} / u, \alpha_{k} \rightarrow \alpha^{\prime}(x), R_{k} \rightarrow R^{\prime}(x), f(x)=$ $\mathrm{d} R^{\prime}(x) / \mathrm{d} x ; v_{k} \rightarrow v^{\prime}(x), T_{k} \rightarrow T^{\prime}(x), \beta_{1}=1 / k_{\mathrm{B}} T^{\prime}(x)$, and proceeding to continuous sampling space, from discrete probabilities to continuous probability density function, and replaced summation by integration and defining density of probability

$$
f\left(\beta_{1} r_{1} / \beta_{0} r_{0}\right)=\frac{\mathrm{d} R^{\prime}(x) / \mathrm{d} x}{\mathrm{~d}\left(\beta_{1} r_{1} / \beta_{0} r_{0}\right) / \mathrm{d} x},
$$

we obtain from (19):

$$
p(E)=\mathrm{e}^{-\beta E} \omega(E) Z^{-1} \int_{0}^{\infty} f\left(y_{1}\right) \mathrm{e}^{-y_{1} \beta_{0} r_{0} E} \mathrm{~d}\left(y_{1}\right) ; \quad \int_{0}^{\infty} f(y) \mathrm{d} y=1 .
$$

Here we assumed that $f\left(\beta_{1} r_{1} / \beta_{0} r_{0}\right)$ is the probability density for a random value $y(x)=\beta(x) r(x) / \beta_{0} r_{0}$ to take on a specific value $y_{1}=\beta_{1} r_{1} / \beta_{0} r_{0}$ under the condition that the system is located at the point $x$ of the potentially possible states considered as a continuous random value (although the initial set of potential values is a discrete set). Like in (18) we wrote $\left(P_{k} v_{k} \alpha_{k}\right) / u=r_{k}$ and represented the exponent in $(21)$ as $-y_{1} \beta_{0} r_{0} E, y_{1}=\left(\beta_{1} r_{1} / \beta_{0} r_{0}\right)$, taking $\beta_{0} r_{0} E$ the argument of the characteristic function. Then the integrand in the r.h.s. of (21) is the Laplas transform for the distribution $f^{\prime}\left(y_{1}\right)$ with argument $\beta_{0} r_{0} E$.

In the open system with complex potential an extra factor $\alpha v \beta$ appears, taking into account the exchange with surrounding (value $\alpha$ ) and the fluctuations of the inverse temperature $\beta$ and values of $P, v, \alpha$. If one performs the replacement of variables $\beta^{\prime}=\beta+r_{1} \beta_{1}$ and assumes that the situation $\beta=0$ is possible, when the bottom limit of integration in (21) after replacement of a variable is 0 , instead of $(21)$ we shall obtain

$$
\begin{aligned}
p(E) & =\omega(E) Z^{-1} \int_{0}^{\infty} f_{1}\left(\beta^{\prime}\right) \exp \left\{-\beta^{\prime} E\right\} \mathrm{d} \beta^{\prime}, \\
f_{1}\left(\beta^{\prime}\right) & =f\left(\beta_{1} r_{1}\right)=f\left[\beta^{\prime}-\beta_{0}\left(1-r_{0}\right)\right],
\end{aligned}
$$

which coincides with superstatistics [1]. But the correlation (21) describes a more general situation and superstatistics forms here a special case (21). The value

$$
\begin{aligned}
& r_{0}=\alpha_{0}\left(P_{0} v_{0} / u\right) ; \quad r_{0}=\langle r\rangle=\int r f(r) \mathrm{d} r ; \quad r_{0}=\langle\alpha P v / u\rangle ; \quad \alpha=\gamma / \lambda, \\
& \alpha_{0}=\int \alpha_{1} f\left(\beta_{1} r_{1}\right) \mathrm{d}\left(\beta_{1} r_{1}\right), \quad r=\alpha / u, \quad P_{0}=\langle P\rangle=\int P_{1} f\left(\beta_{1} r_{1}\right) \mathrm{d}\left(\beta_{1} r_{1}\right) .
\end{aligned}
$$

For ideal gas $P / u \approx 0,687$. It is possible to mention some examples of physical realization of distribution (21). So, for a case of superdiffusion in [33] at the absence of external force, stationary distribution is in Tsallis form, and for a case of the presence of constant external force, the multiplicative noise in exponential form of distribution is manifested. As in (21), two kinds of distributions are combined in a single phenomenon for one system. The obtained distribution of the kind (21) provides ample opportunities for describing various physical situations.

In [34] it is shown that the temperature fluctuations can be described using the gammadistribution. As in [1] for distribution function $f$ in (21) similar to the gamma-distribution in (15) (but with variables $\alpha_{k}=c, b_{k}=b ; f(y)=\Gamma^{-1}(c) b^{-c}(y)^{c-1} \exp \{-y / b\}$ ) expression (21) reduces to the product of Gibbs distribution and Tsallis distribution of a kind $(c=1 /(q-1) ; b=q-1)$

$$
p(E)=\mathrm{e}^{-\beta E} \omega(E) Z^{-1}(1+b E)^{-c}=\mathrm{e}^{-\beta E} \omega(E) Z^{-1}\left[1+(q-1) \beta_{0} r_{0} E\right]^{-1 /(q-1)} .
$$

At $q \rightarrow 1$ one gets from (22) the expression $\exp \left\{-\beta_{0} E\right\}$, which at $r_{0}=0$ yields Gibbs distribution $\exp \{-\beta E\}$. In $(22)$ we can separate the Tsallis temperature, $\beta_{q}=\beta_{\mathrm{Ts}}=\beta r_{0} /\left(1-r_{0}\right)=\beta_{0} r_{0}$. Thus 
we do have three different "temperatures": Gibbs temperature $(7) \beta=\beta_{\text {Gibbs }}=1 / k_{\mathrm{B}} T=\beta_{0}\left(1-r_{0}\right)$; effective temperature $\beta_{0}=\beta_{\text {eff }}=\beta /\left(1-r_{0}\right)$; and Tsallis temperature $\beta_{q}=\beta_{\mathrm{Ts}}=\beta_{0} r_{0}=\beta r_{0} /(1-$ $\left.r_{0}\right)$. The following relations between them hold: $\beta_{0}=\beta+\beta_{q} ; r_{0}=1-\beta / \beta_{0}=\beta_{q} / \beta_{0}$. It is obvious that distribution (21) represents the product of Boltzmann factor with a multiplier superstatistics from which Tsallis distribution as a special case can be obtained (having chosen $f$ in $(21)$ the gamma-distribution of a kind (15)). The behaviour of distribution (21), (22) essentially depends on the values $r_{0}\left(\alpha_{0}\right)$. At $r_{0} \rightarrow 0\left(\alpha_{0} \rightarrow 0, \alpha_{0}>0\right)$ the Gibbs distribution is obtained. At $\alpha \rightarrow 0$ and $\gamma \rightarrow 0$. As it is marked in the beginning of section 3, it corresponds to transition to Gibbs distributions. Thus, not only $\lambda \rightarrow 0$, but also $\alpha=\gamma / \lambda \rightarrow 0$. The value $\gamma$ describes entropy fluxes and entropy production in the system [15]; the value $\lambda$ describes the intensity of an input in the system (and an output from it). Similarly to the discrete case, one can specify the expressions obtained. As $f$ in (21) one can choose various continuous expressions.

The distributions (21), (22) are widely encountered in various domains of physics. For example, for distributions in the systems with multiplicative noise in [35] the stationary solution is written as

$$
p(u)=C / \eta(u) ; \quad C=\text { const; } \quad \eta(u)=\left(M g^{2}+A\right)^{1 / 2} \mathrm{e}^{-\int h(u) \mathrm{d} u /\left(M g^{2}+A\right)},
$$

where $\mathrm{d} u / \mathrm{d} t=h(u)+g(u) \xi(t)+\eta(t), u(t)$ is a stochastic variable, $h, g$ are arbitrary functions $(g(0)=0)$, and $\xi(t), \eta(t)$ are uncorrelated and Gaussian-distributed zero-mean white noises, hence satisfying $\left\langle\xi(t) \xi\left(t^{\prime}\right)\right\rangle=2 M \delta\left(t-t^{\prime}\right),\left\langle\eta(t) \eta\left(t^{\prime}\right)\right\rangle=2 A \delta\left(t-t^{\prime}\right)$, where $M, A>0$ are the noise amplitudes and stand for multiplicative and additive, respectively. If we assume the deterministic drift $h(u)$ and $g(u)$ in the form widely used in synergetics (for example, [36]): $h(u)=\alpha u-$ $\delta u^{3} ; g(u)=u$, then from $(23)$ :

$$
p(u) \sim \mathrm{e}^{-\delta u^{2} / 2 M} /\left[1+M u^{2} / A\right]^{[1-(\alpha+\delta A / M) / M] / 2} .
$$

Comparing (24) and (22), we get

$$
\begin{aligned}
& E=u^{2} / 2 ; \quad \delta / M=\beta=1 / k_{\mathrm{B}} T ; \quad 1 /(q-1)=[1-(\alpha+\delta A / M) / M] / 2 \\
& (q-1) \beta_{0} r_{0}=M / A ; \quad r_{0}=1-\delta A / M(M-\alpha)=1-\beta A /(M-\alpha) ; \\
& \beta_{0}=\beta /\left(1-r_{0}\right)=\beta+\beta_{q}=(M-\alpha) / A ; \\
& \beta_{q}=\beta_{0} r_{0}=(M-\alpha) / A-\beta=\beta_{0}-\beta .
\end{aligned}
$$

Hence, it is clear that the sign of $q-1, r_{0}$ depends on the relation between $\alpha, \delta, A, M$. The value $\beta_{0}=\beta$ at $\alpha=M-\beta A$. The value $q-1$ has a singularity at $1=(\alpha+\delta A / M) / M$, changing its sign. At $\alpha=M$ the value $\beta_{0}$ turns to zero and the value $r_{0}$ has a singular behaviour. It follows that the value of the controlling parameter $\alpha=M$ corresponds to the bifurcation point. Besides the (24) one can name a number of different examples of choosing functions $h(u)$ and $g(u)[30,35]$. Not only gamma-distributions (yielding Tsallis distribution (22)), but also other possible classes of functions can be used as $f$ in (21).

Turbulence, Van der Pol generators, the velocity distribution in the presence of the heat source, space diffusion of the Brownian particles in the external field, Maltus-Ferhuelst process, Van der Pol-Duffing system [24] and many examples of distributions of other phenomena (self-organized criticality, a spectrum of the cosmic ray) which lead to expressions of the kind (21), (22) are described in the same way.

\section{Conclusion}

From the distribution (5) containing lifetime of statistical system (first-passage time, time before degeneration of system) we obtained the energy distributions of a system which contain the product of the Gibbs factor and a factor corresponding to the superstatistics. In the superstatistics factor one averages not only over the values of the finite temperature, but also over other system parameters, including the parameters of interaction with the environment.

The superstatistics theory $[1,2]$ is the generalization of the nonextensive statistical mechanics $[3,4]$. Some of the fields of applications of the nonextensive statistical mechanics are metastable 
states, Levy flights, systems of finite size [37]. All these phenomena are conditioned physically by the finiteness of the lifetime, and by the possibilities of small probability big fluctuations which are generally dismissed when accounting for the system evolution.

The introduction of the lifetime (first-passage time) as a thermodynamic parameter is justified by the fact that finite systems possess finite lifetimes, which essentially effects their properties and the properties of their environment. The lifetime is interpreted as a fundamental quality having dual nature related to both the flux of external time, and the properties of a system. In [15] the expressions for the lifetime through dynamical characteristics of a system were obtained. These expressions show the dependence on the interchange with the environment.

The Gibbs distribution does not describe the dissipative processes that develop in the system. Superstatistics describe systems by constantly putting the energy into the system, which is dissipated. The value $\alpha=\gamma / \lambda$ is related to dissipative processes in the system (through $\gamma$ ). It defines a correlation between Gibbs and superstatistics multipliers in the distribution (21). The value $\gamma$ thermodynamically conjugated to random lifetime is expressed through entropy production and currents $[7,15]$, i.e. through communication of the system with an the environment. The obtained distribution contains a new parameter related to a thermodynamic state of the system, as well as with distribution of a lifetime of metastable states and interaction of these states with the environment. Changing this parameter it is possible to go over to Gibbs distribution.

Thus, the lifetime finiteness being explicitly taken into account for a statistical system and the use of the lifetime (first-passage time) as a thermodynamic variable brings us to the nonextensive statistical mechanics. This fact is in correspondence with the known results regarding the finiteness of the size for systems in which the nonextensive statistical mechanics can be applied [37]. Furthermore we obtained the distribution generalizing the Gibbs-Boltzmann statistics and superstatistics. Real open systems seem to share the properties of both Gibbs and nonextensive statistical mechanics. So we believe such kinds of distributions to be a promissing point of view, which is also confirmed by the relevance of such distributions to various phenomena. Depending on the value of $r_{0}$ and on the controlling parameter, the properties of either nonextensive or extensive statistical mechanics prevail. The obtained relation (21) can be regarded as a detailed elaboration of the superstatistics theory [1]. For the expression (21) one can write the entropy expression as well as the corresponding thermodynamic relations.

\section{References}

1. Beck C., Cohen E.G.D., Physica A, 2003, 322A, 267; Cond-Mat/0205097.

2. Cohen E.G.D., Physica D, 2004, 193D, 35.

3. Tsallis C., J. Stat. Phys. 1988, 52, 479.

4. http://tsallis.cat.cbpf.br/biblio.htm

5. Ryazanov V.V., Shpyrko S.G. Reports of Institute for Nuclear Research. Kiev, 1998, 117 (in Russian).

6. Ryazanov V.V., Shpyrko S.G. Reports of Institute for Nuclear Research. Kiev, 1999, 155 (in Russian).

7. Ryazanov V.V., Shpyrko S.G. Nonequilibrium Thermodynamics and Lifetime of Physical Systems. Preprint Cond-Mat/0406359, 2004.

8. Ryazanov V.V. Superstatistics and Lifetime. Preprint Cond-Mat/0404357, 2004.

9. Stratonovich R.L. The elected questions of the fluctuations theory in a radio engineering. Gordon and Breach, New York, 1967.

10. Pontryagin L.A., Andronov A.A., Vitt A.A., Zh. Eksp. Teor. Fiz., 1933, 3, 165 (translated by J.B.Barbour and reproduced in "Noise in Nonlinear Dynamics", 1989, edited by F.Moss and P.V.E.McClintock. Cambridge University Press, Cambridge, 1, 329).

11. Kramers H.A., Physica, 1940, 7, 284.

12. Van Kampen N.G. Stochastic Processes in Physics and Chemistry. North-Holland, Amsterdam, 1992.

13. Gardiner C.W. Handbook of Stochastic Methods, 2nd edition. Springer, Berlin, 1990.

14. Leontovich M.A. Introduction to Thermodynamics. Statistical Physics, Nauka, Moscow, 1985 (in Russian).

15. Ryazanov V.V., Fortschritte der Phusik/Progress of Physics, 2001, 49, 885.

16. Zubarev D.N. Nonequilibrium Statistical Thermodynamics. Consultants Bureau, New York, 1974. 
17. Zubarev D.N. In Reviews of Science and Technology: Modern Problems of Mathematics. Vol. 15, 131-226, (in Russian), ed. by R.B.Gamkreidze. Nauka, Moscow, 1980

18. Luzzi R., Vasconcellos A.R., Ramos J.G., International Journal of modern physics B, 2000, 14, 3189.

19. Kirkwood J.G., J. Chem. Phys., 1946, 14, 180; 1946, 15, 72.

20. Ginzburg S.L., Zh. Eksp. Teor. Fiz., 1986, 90, 754.

21. Naudts J., Czachor M. Thermodynamic origin of universal fluctuations and two-power laws. Preprint Cond-Mat/0108354, 2001.

22. Feller W. An Introduction to Probability Theory and its Applications, 2. J. Wiley, New York, 1971.

23. Prabhu N.U. Stochastic Storage Processes. Springer Verlag, New York, 1980.

24. Klimontovich Yu.L. Statistical Theory of Open Systems. Kluwer Acad. Publ., Dordrecht, 1995.

25. Jaynes E.T., Phys. Rev., 1957, 106, 620; 1957, 108, 171.

26. Korolyuk V.S., Turbin A.F. Mathematical Foundations of the State Lumping of Large Systems. Kluwer Acad. Publ., Dordrecht, Boston/London, 1993.

27. Gaspard P., Nicolis G., Phys. Rev. Lett., 1990, 65, (14), 1693.

28. Dorfman J.R., Gaspard P., Phys.Rev. E, 1995, 51, 28.

29. Gaspard P., Dorfman J.R., Phys. Rev. E, 1995, 52, 3525.

30. Olemskoi A.I., Physics-Uspekhi, 1993, 163, 1.

31. Ryazanov V.V., Ukrainian Journal of Physics, 1993, 38, 615.

32. Klimontovich Yu.L. Statistical Physics. Yarwood Acadenic Publishers, New-York, 1984.

33. Olemskoi A.I., Kharchenko D.A., Journal of Physical Studies, 2002, 6, 253.

34. Lavenda B.H. On the definition of fluctuating temperature. Preprint Cond-Mat/0401024, 2004.

35. Anteneodo C., Tsallis C., Multiplicative noise: A mechanism leading to nonextensive statistical mechanic. J. Math. Phys., 2003, 44, 5194; Cond-Mat/0205314.

36. Haken H. Synergetics, An Introducton. Springer, Berlin, 1983.

37. Potiguar F.Q., Costa U.M.S., Fluctuation of energy in the generalized thermostatistics. Physica A, 2003, 321, 482; Cond-Mat/0210525.

\title{
Час першого проходження: поняття, що приводить до суперстатистик
}

\author{
В.В.Рязанов, С.Г.Шпирко \\ Інститут ядерних досліджень НАН України, \\ Київ, пр. Науки, 47
}

Отримано 14 липня 2005 р.

Для опису нерівноважних станів системи вводиться новий термодинамічний параметр - час життя системи (час першого досягнення певної границі або рівня). Записуються статистичні розподіли, які можна одержати з мезоскопічного опису, що характеризує систему при специфікуванні стохастичного процесу. Суперстатистики (і їхні узагальнення), що вводяться в [Beck C., Cohen E.G.D., Physica A, 2003, 322A, 267], отримані зі статистичного розподілу з часом життя, як термодинамічним параметром.

Ключові слова: стаціонарні стани, стохастичні процеси, неканонічні статистичні розподіли

PACS: $05.70 . \mathrm{Ln}, 05.40 .-\mathrm{a}, 05.10 . \mathrm{Gg}, 05.20 . \mathrm{Gg}$ 